Proceedings of the Institute of Mathematics and Mechanics,

National Academy of Sciences of Azerbaijan

Volume 47, Number 1, 2021, Pages 3-14

https://doi.org/10.30546/2409-4994.47.1.3

\title{
EXISTENCE AND UNIQUENESS OF SOLUTIONS FOR A NABLA FRACTIONAL BOUNDARY VALUE PROBLEM WITH DISCRETE MITTAG-LEFFLER KERNEL
}

\author{
JAGAN MOHAN JONNALAGADDA AND DUMITRU BALEANU
}

\begin{abstract}
We consider a two-point boundary-value problem of order $1<\alpha<\frac{3}{2}$ involving nabla fractional differences with discrete MittagLeffler kernels. In [2], the authors obtained an expression for the Green's function of this boundary value problem. We determine an upper bound for the Green's function and derive a Lyapunov-type inequality. Further, we also establish sufficient conditions on existence and uniqueness of solutions for the corresponding nonlinear problem using fixed point theorems.
\end{abstract}

\section{Introduction}

Nabla fractional calculus [9] is the theory of arbitrary order sum and difference operators in the backward sense. Since nabla fractional sums and differences can be used to model nonlocal phenomena, the theory of nabla fractional difference equations has become a fruitful field of research in science and engineering. For applications of nabla fractional calculus, we refer the reader to $[4,7,15,18,19$, $20,21]$ and the references therein.

The dynamics of many real world phenomena in nature change only at discrete times. If a natural system could be modeled by a discrete time system, many qualitative aspects of such a system could be determined. But, these discrete phenomena cannot be described by a single type of difference or fractional difference operator. Therefore, new discrete operators and their properties, particularly nabla fractional difference operators, have to be analyzed and investigated. The nabla discrete Mittag-Leffler function arises naturally in the solutions of nabla fractional difference equations, and especially in investigations of discrete fractional generalizations of the kinetic equation, random walks, Levy flights, super-diffusive transport, and so on. Motivated by these facts, recently, several authors introduced new nabla fractional difference operators with discrete exponential [3] and discrete Mittag-Leffler kernels [1, 2, 14], and studied their real world applications.

In this article, we consider a two-point boundary-value problem of order $1<$ $\alpha<\frac{3}{2}$ involving nabla fractional differences with discrete Mittag-Leffler kernels,

2010 Mathematics Subject Classification. 26A33, 39A12, 39A70.

Key words and phrases. Nabla fractional difference, discrete Mittag-Leffler kernel, fixed point, existence, uniqueness. 
and derive a Lyapunov-type inequality using the properties of its Green's function. Further, we also establish sufficient conditions on existence and uniqueness of solutions for the corresponding nonlinear problem using Brouwer and Banach fixed point theorems.

\section{Preliminaries}

The following preliminaries shall be used in this article. Let $a, b \in \mathbb{R}$ with $b-a \in \mathbb{N}_{1}$. Represent by $\mathbb{N}_{a}:=\{a, a+1, a+2, \ldots\}$ and $\mathbb{N}_{a}^{b}:=\{a, a+1, a+2, \ldots, b\}$. The jump operator in the backward sense $\rho$ is defined by

$$
\rho(t):=t-1, \quad t \in \mathbb{N}_{a+1} .
$$

Definition 2.1 (See [9]). For $t \in \mathbb{R} \backslash\{\cdots,-2,-1,0\}$ and $r \in \mathbb{R}$ such that $(t+r) \in \mathbb{R} \backslash\{\cdots,-2,-1,0\}$, the generalized rising function is defined by

$$
t^{\bar{r}}:=\frac{\Gamma(t+r)}{\Gamma(t)}, \quad 0^{\bar{r}}:=0
$$

Here $\Gamma(\cdot)$ denotes the Euler gamma function.

Definition 2.2 (See [9]). The $\mu^{t h}$-order nabla fractional Taylor monomial is defined by

$$
H_{\mu}(t, a)=\frac{(t-a)^{\bar{\mu}}}{\Gamma(\mu+1)}, \quad \mu \in \mathbb{R} \backslash\{\ldots,-2,-1\} .
$$

Definition 2.3 (See [9]). Let $\nu>0$ and $u: \mathbb{N}_{a+1} \rightarrow \mathbb{R}$. The $\nu^{\text {th }}$-order nabla sum of $u$ is defined by

$$
\left(\nabla_{a}^{-\nu} u\right)(t)=\sum_{s=a+1}^{t} H_{\nu-1}(t, \rho(s)) u(s), \quad t \in \mathbb{N}_{a} .
$$

Notice that $\left(\nabla_{a}^{-\nu} u\right)(a)=0$.

Definition 2.4 (See [9]). Let $\lambda \in \mathbb{R}$ and $\alpha, \beta, z \in \mathbb{C}$ such that $|\lambda|<1$ and $\Re(\alpha)>0$. The nabla discrete Mittag-Leffler functions are defined by

$$
E_{\overline{\alpha, \beta}}(\lambda, z):=\sum_{k=0}^{\infty} \lambda^{k} \frac{z^{\overline{k \alpha+\beta-1}}}{\Gamma(k \alpha+\beta)}
$$

and

$$
E_{\bar{\alpha}}(\lambda, z):=E_{\overline{\alpha, 1}}(\lambda, z):=\sum_{k=0}^{\infty} \lambda^{k} \frac{z^{\overline{k \alpha}}}{\Gamma(k \alpha+1)} .
$$

Definition 2.5 (See [1]). Let $u: \mathbb{N}_{a} \rightarrow \mathbb{R}$ and $0<\nu<\frac{1}{2}$. The $\nu^{\text {th }}$-order Caputo-type nabla difference with discrete Mittag-Leffler kernel is defined by

$$
\left(\begin{array}{l}
A B C \\
a
\end{array} \nabla^{\nu} u\right)(t):=\frac{B(\nu)}{1-\nu} \sum_{s=a+1}^{t}(\nabla u)(s) E_{\bar{\nu}}\left(-\frac{\nu}{1-\nu}, t-\rho(s)\right), \quad t \in \mathbb{N}_{a+1} .
$$

The corresponding nabla sum is given by

$$
\left({ }_{a}^{A B} \nabla^{-\nu} u\right)(t):=\frac{1-\nu}{B(\nu)} u(t)+\frac{\nu}{B(\nu)}\left(\nabla_{a}^{-\nu} u\right)(t), \quad t \in \mathbb{N}_{a+1} .
$$

Here $B(\nu)>0$ is a normalization function satisfying $B(0)=B(1)=1$. 
Definition 2.6 (See [2]). Let $u: \mathbb{N}_{a-1} \rightarrow \mathbb{R}$ and $1<\nu<\frac{3}{2}$. The $\nu^{\text {th }}$-order Caputo-type nabla difference with discrete Mittag-Leffler kernel is defined by

$$
\left({ }_{a}^{A B C} \nabla^{\nu} u\right)(t):=\left({ }_{a}^{A B C} \nabla^{\nu-1}(\nabla u)\right)(t), \quad t \in \mathbb{N}_{a+1}
$$

The corresponding nabla sum is given by

$$
\left({ }_{a}^{A B} \nabla^{-\nu} u\right)(t):=\frac{2-\nu}{B(\nu-1)}\left(\nabla_{a}^{-1} u\right)(t)+\frac{\nu-1}{B(\nu-1)}\left(\nabla_{a}^{-\nu} u\right)(t), \quad t \in \mathbb{N}_{a+1}
$$

Remark 2.1. For $0<\nu<1$, the parameter $\lambda=-\frac{\nu}{1-\nu}$ in the discrete MittagLeffler kernel satisfies $|\lambda|<1$ for the convergence purpose if and only if $0<\nu<\frac{1}{2}$. Similarly, for $1<\nu<2$, the parameter $\lambda=-\frac{\nu-1}{2-\nu}$ in the discrete Mittag-Leffler kernel satisfies $|\lambda|<1$ for the purpose of convergence if and only if $1<\nu<\frac{3}{2}$.

Proposition 2.1 (See [10]). Let $s \in \mathbb{N}_{a}$ and $-1<\mu$. The following properties hold:

(a) $H_{\mu}(t, \rho(s)) \geq 0$ for $t \in \mathbb{N}_{\rho(s)}$, and if $t \in \mathbb{N}_{s}$, then $H_{\mu}(t, \rho(s))>0$.

(b) $H_{\mu}(t, \rho(s))$ is a decreasing function with respect to $s$ for $t \in \mathbb{N}_{\rho(s)}$ and $\mu \in$ $(0, \infty)$, and if $t \in \mathbb{N}_{s}$ and $\mu \in(-1,0)$, then $H_{\mu}(t, \rho(s))$ is an increasing function of $s$.

(c) $H_{\mu}(t, \rho(s))$ is a nondecreasing function with respect to $t$ for $t \in \mathbb{N}_{\rho(s)}$ and $\mu \in[0, \infty)$, and if $t \in \mathbb{N}_{s}$ and $\mu \in(0, \infty)$, then $H_{\mu}(t, \rho(s))$ is an increasing function of $t$.

(d) $H_{\mu}(t, \rho(s))$ is a decreasing function with respect to $t$ for $t \in \mathbb{N}_{s+1}$ and $\mu \in(-1,0)$.

Proposition 2.2 (See [10]). Let $u$ and $v$ be two nonnegative real-valued functions defined on a set $S$. Further, assume $u$ and $v$ achieve their maximum values in $S$. Then,

$$
|u(t)-v(t)| \leq \max \{u(t), v(t)\} \leq \max \left\{\max _{t \in S} u(t), \max _{t \in S} v(t)\right\},
$$

for every fixed $t$ in $S$.

\section{Lyapunov-type Inequality}

Assume $1<\alpha<\frac{3}{2}$ and $h: \mathbb{N}_{a+1}^{b} \rightarrow \mathbb{R}$. Consider the boundary-value problem

$$
\left\{\begin{array}{l}
\left(\begin{array}{l}
A B C \\
a
\end{array} \nabla^{\alpha} y\right)(t)+h(t)=0, \quad t \in \mathbb{N}_{a+1}^{b-1}, \\
y(\rho(a))=y(b)=0 .
\end{array}\right.
$$


In [2], the authors obtained the following expression for the unique solution of $(3.1)$.

$$
\begin{aligned}
y(t) & =\frac{(2-\alpha)(t-\rho(a))}{(b-\rho(a)) B(\alpha-1)} \sum_{s=a+1}^{b} h(s) \\
+ & \frac{(\alpha-1)(t-\rho(a))}{(b-\rho(a)) B(\alpha-1)} \sum_{s=a+1}^{b} H_{\alpha-1}(b, \rho(s)) h(s)-\frac{(2-\alpha)}{B(\alpha-1)} \sum_{s=a+1}^{t} h(s) \\
& \quad-\frac{(\alpha-1)}{B(\alpha-1)} \sum_{s=a+1}^{t} H_{\alpha-1}(t, \rho(s)) h(s), \quad t \in \mathbb{N}_{\rho(a)}^{b} .
\end{aligned}
$$

In Theorem 3.1, we rearrange this solution and determine the expression for the Green's function.

Theorem 3.1. [2] The unique solution of (3.1) is given by

$$
y(t)=\sum_{s=a+1}^{b} G(t, s) h(s), \quad t \in \mathbb{N}_{\rho(a)}^{b},
$$

where

$$
\begin{gathered}
G(t, s)= \begin{cases}G_{1}(t, s), & t \in \mathbb{N}_{\rho(a)}^{s-1}, \\
G_{2}(t, s), & t \in \mathbb{N}_{s}^{b}\end{cases} \\
G_{1}(t, s)=\frac{(t-\rho(a))}{(b-\rho(a)) B(\alpha-1)}\left[(2-\alpha)+(\alpha-1) H_{\alpha-1}(b, \rho(s))\right],
\end{gathered}
$$

and

$$
G_{2}(t, s)=G_{1}(t, s)-\frac{1}{B(\alpha-1)}\left[(2-\alpha)+(\alpha-1) H_{\alpha-1}(t, \rho(s))\right] .
$$

Remark 3.1. Clearly, $G(\rho(a), s)=G_{1}(\rho(a), s)=0$ and $G(b, s)=G_{2}(b, s)=0$.

Lemma 3.1. For all $(t, s) \in \mathbb{N}_{\rho(a)}^{b} \times \mathbb{N}_{a+1}^{b}$,

$$
|G(t, s)| \leq \frac{1}{B(\alpha-1)}\left[(2-\alpha)+(\alpha-1) H_{\alpha-1}(b, a)\right]
$$

Proof. Assume $t \in \mathbb{N}_{\rho(a)}^{s-1}$ and $s \in \mathbb{N}_{a+1}^{b}$. Clearly, $\frac{(t-\rho(a))}{(b-\rho(a)) B(\alpha-1)} \geq 0,(2-\alpha) \geq 0$ and $(\alpha-1)>0$. From Proposition 2.1, we have $H_{\alpha-1}(b, \rho(s))>0$. Thus,

$$
G_{1}(t, s)>0, \quad t \in \mathbb{N}_{\rho(a)}^{s-1}, \quad s \in \mathbb{N}_{a+1}^{b} .
$$

Since $(t-\rho(a)) \leq(b-1-\rho(a))$ and, from Proposition 2.1, we have $H_{\alpha-1}(b, \rho(s)) \leq$ $H_{\alpha-1}(b, a)$, it follows that

$$
\left|G_{1}(t, s)\right| \leq \frac{(b-a)}{(b-\rho(a)) B(\alpha-1)}\left[(2-\alpha)+(\alpha-1) H_{\alpha-1}(b, a)\right] .
$$

Now, assume $t \in \mathbb{N}_{s}^{b}$ and $s \in \mathbb{N}_{a+1}^{b}$. Consider

$$
\left|G_{2}(t, s)\right|=\left|G_{1}(t, s)-\tilde{G}(t, s)\right|,
$$


where

$$
\tilde{G}(t, s)=\frac{1}{B(\alpha-1)}\left[(2-\alpha)+(\alpha-1) H_{\alpha-1}(t, \rho(s))\right] .
$$

From Proposition 2.1, we have $H_{\alpha-1}(b, \rho(s)), H_{\alpha-1}(t, \rho(s))>0$. Consequently, $G_{1}(t, s), \tilde{G}(t, s)>0$. Then, by Proposition 2.2 , we obtain

$$
\left|G_{2}(t, s)\right| \leq \max \left\{\max _{t \in \mathbb{N}_{s}^{b}, s \in \mathbb{N}_{a+1}^{b}} G_{1}(t, s), \max _{t \in \mathbb{N}_{s}^{b}, s \in \mathbb{N}_{a+1}^{b}} \tilde{G}(t, s)\right\} .
$$

Clearly, $(t-\rho(a)) \leq(b-\rho(a))$ and, from Proposition 2.1, we have

(i) $H_{\alpha-1}(b, \rho(s)) \leq H_{\alpha-1}(b, a)$;

(ii) $H_{\alpha-1}(t, \rho(s)) \leq H_{\alpha-1}(b, a)$.

Thus, we have

$$
\max _{t \in \mathbb{N}_{s}^{b}, s \in \mathbb{N}_{a+1}^{b}} G_{1}(t, s)=\frac{1}{B(\alpha-1)}\left[(2-\alpha)+(\alpha-1) H_{\alpha-1}(b, a)\right],
$$

and

$$
\max _{t \in \mathbb{N}_{s}^{b}, s \in \mathbb{N}_{a+1}^{b}} \tilde{G}(t, s)=\frac{1}{B(\alpha-1)}\left[(2-\alpha)+(\alpha-1) H_{\alpha-1}(b, a)\right] .
$$

Consequently,

$$
\left|G_{2}(t, s)\right| \leq \frac{1}{B(\alpha-1)}\left[(2-\alpha)+(\alpha-1) H_{\alpha-1}(b, a)\right] .
$$

Therefore, from (3.7) and (3.8), we obtain the required result. The proof is complete.

Now, we derive a Lyapunov-type inequality for

$$
\left\{\begin{array}{l}
\left(\begin{array}{l}
A B C \\
a
\end{array} \nabla^{\alpha} y\right)(t)+q(t) y(t)=0, \quad t \in \mathbb{N}_{a+1}^{b-1}, \\
y(\rho(a))=y(b)=0
\end{array}\right.
$$

where $q: \mathbb{N}_{a+1}^{b} \rightarrow \mathbb{R}$.

Theorem 3.2. If (3.9) possess a nontrivial solution, then

$$
\sum_{s=a+1}^{b}|q(s)| \geq \frac{B(\alpha-1)}{\left[(2-\alpha)+(\alpha-1) H_{\alpha-1}(b, a)\right]} .
$$

Proof. Use the norm

$$
\|y\|=\max _{t \in \mathbb{N}_{\rho(a)}^{b}}|y(t)| .
$$

We know that every solution of (3.9) satisfies the following equation:

$$
y(t)=\sum_{s=a+1}^{b} G(t, s) q(s) y(s) .
$$


Consider

$$
\begin{aligned}
|y(t)| & \leq \sum_{s=a+1}^{b}|G(t, s)||q(s)||y(s)| \\
& \leq\|y\| \sum_{s=a+1}^{b}|G(t, s)||q(s)| \\
& \leq \frac{\|y\|}{B(\alpha-1)}\left[(2-\alpha)+(\alpha-1) H_{\alpha-1}(b, a)\right] \sum_{s=a+1}^{b}|q(s)|,
\end{aligned}
$$

implying that (3.10) holds.

\section{Existence \& Uniqueness Results}

We present some existence and uniqueness results for the following boundary value problem using various fixed point theorems.

$$
\left\{\begin{array}{l}
-\left(\begin{array}{l}
A B C \\
a
\end{array} \nabla^{\alpha} y\right)(t)=f(t, y(t)), \quad t \in \mathbb{N}_{a+1}^{b-1}, \\
y(\rho(a))=y(b)=0,
\end{array}\right.
$$

where $1<\alpha<\frac{3}{2}$ and $f: \mathbb{N}_{a+1}^{b} \times \mathbb{R} \rightarrow \mathbb{R}$. By Theorem 3.1, we observe that $y$ is a solution of (4.1) if and only if $y$ is a solution of the summation equation

$$
y(t)=\sum_{s=a+1}^{b} G(t, s) f(s, y(s)), \quad t \in \mathbb{N}_{\rho(a)}^{b} .
$$

Any solution $y: \mathbb{N}_{\rho(a)}^{b} \rightarrow \mathbb{R}$ of (4.1) can be treated as a real $(b-a+2)$-tuple vector. Consequently, $y \in \mathbb{R}^{b-a+2}$. Define the operator $T: \mathbb{R}^{b-a+2} \rightarrow \mathbb{R}^{b-a+2}$ by

$$
(T y)(t)=\sum_{s=a+1}^{b} G(t, s) f(s, y(s)), \quad t \in \mathbb{N}_{\rho(a)}^{b} .
$$

Clearly, $y$ is a fixed point of $T$ if and only if $y$ is a solution of (4.1). We use the fact that $\mathbb{R}^{b-a+2}$ is a Banach space equipped with the maximum norm defined by

$$
\|y\|=\max _{t \in \mathbb{N}_{\rho(a)}^{b}}|y(t)|
$$

Let

be a closed ball in $\mathbb{R}^{b-a+2}$.

$$
\mathcal{B}_{R}=\left\{y \in \mathbb{R}^{b-a+2}:\|y\| \leq R\right\}
$$

\subsection{Assumptions. Assume}

(C 1) $f: \mathbb{N}_{a+1}^{b} \times \mathbb{R} \rightarrow \mathbb{R}$ is continuous;

(C 2) $|f(t, y)| \leq M$ for all $(t, y) \in \mathbb{N}_{a+1}^{b} \times \mathcal{B}_{R}$;

(C 3) $f$ is bounded on $\mathbb{N}_{a+1}^{b} \times \mathbb{R}^{b-a+2}$;

(C 4) For all $(t, y),(t, z) \in \mathbb{N}_{a+1}^{b} \times \mathcal{B}_{R}$,

$$
\|f(t, y)-f(t, z)\| \leq K\|y-z\| .
$$

(C 5) $\max _{t \in \mathbb{N}_{a+1}^{b}}|f(t, 0)|=P$ and $\max _{(t, y) \in \mathbb{N}_{a+1}^{b} \times \mathcal{B}_{R}}|f(t, y)|=Q$; 
(C 6) $\frac{K}{B(\alpha-1)}\left[(2-\alpha)+(\alpha-1) H_{\alpha-1}(b, a)\right]<1$;

(C 7) For all $(t, y),(t, z) \in \mathbb{N}_{a+1}^{b} \times \mathbb{R}^{b-a+2}$,

$$
\|f(t, y)-f(t, z)\| \leq L\|y-z\| .
$$

(C 8) $\frac{L}{B(\alpha-1)}\left[(2-\alpha)+(\alpha-1) H_{\alpha-1}(b, a)\right]<1$.

4.2. Existence of Solutions. First, we apply Brouwer fixed point theorem to establish existence of solutions of (4.1).

Theorem 4.1. [5] (Brouwer fixed point theorem) Let $C$ be a nonempty bounded closed convex subset of $\mathbb{R}^{n}$ and $T: C \rightarrow C$ be a continuous mapping. Then, $T$ has a fixed point in $C$.

Theorem 4.2. (Local Existence) Assume (C 1) and (C 2) hold. If we choose

$$
R \geq \frac{M}{B(\alpha-1)}\left[(2-\alpha)+(\alpha-1) H_{\alpha-1}(b, a)\right],
$$

then (4.1) has a solution in $\mathcal{B}_{R}$.

Proof. We claim that $T: \mathcal{B}_{R} \rightarrow \mathcal{B}_{R}$. To see this, let $y \in \mathcal{B}_{R}, t \in \mathbb{N}_{\rho(a)}^{b}$ and consider

$$
\begin{aligned}
|(T y)(t)| & \leq \sum_{s=a+1}^{b}|G(t, s)||f(s, y(s))| \\
& \leq M \sum_{s=a+1}^{b}|G(t, s)| \\
& \leq \frac{M}{B(\alpha-1)}\left[(2-\alpha)+(\alpha-1) H_{\alpha-1}(b, a)\right] \leq R,
\end{aligned}
$$

implying that $T: \mathcal{B}_{R} \rightarrow \mathcal{B}_{R}$. Since $f$ is continuous, $T$ is also continuous. Hence, (4.1) has at least one solution $y$ in $\mathcal{B}_{R}$ by Theorem 4.1. The proof is complete.

Theorem 4.3. (Global Existence) Assume (C 1) and (C 3) hold. Then, (4.1) has a solution in $\mathbb{R}^{b-a+2}$.

Proof. The proof is similar to the proof of Theorem 4.2.

4.3. Existence \& Uniqueness Results. Next, we use Banach's theorem to establish existence and uniqueness of solutions of (4.1).

Theorem 4.4. [5] (Banach fixed point theorem) Let $\mathcal{B}_{r}$ be the closed ball of radius $r>0$, centered at zero, in $\mathbb{R}^{n}$ with $T: \mathcal{B}_{r} \rightarrow \mathbb{R}^{n}$ a contraction mapping and $T\left(\partial \mathcal{B}_{r}\right) \subseteq \mathcal{B}_{r}$. Then, $T$ has a unique fixed point in $\mathcal{B}_{r}$.

Theorem 4.5. [5] (Banach fixed point theorem) Let $T: \mathbb{R}^{n} \rightarrow \mathbb{R}^{n}$ be a contraction. Then, $T$ possesses a unique fixed point in $\mathbb{R}^{n}$.

Theorem 4.6. (Local Existence \& Uniqueness) Assume (C 1), (C 4), (C 5) and (C 6) hold. If we choose

$$
R \geq \frac{\frac{P}{B(\alpha-1)}\left[(2-\alpha)+(\alpha-1) H_{\alpha-1}(b, a)\right]}{1-\frac{K}{B(\alpha-1)}\left[(2-\alpha)+(\alpha-1) H_{\alpha-1}(b, a)\right]},
$$


or

$$
R \geq \frac{Q}{B(\alpha-1)}\left[(2-\alpha)+(\alpha-1) H_{\alpha-1}(b, a)\right]
$$

then (4.1) has a unique solution in $\mathcal{B}_{R}$.

Proof. Clearly, $T: \mathcal{B}_{R} \rightarrow \mathbb{R}^{b-a+2}$. Now, we claim that $T$ is a contraction. To see this, let $y, z \in \mathcal{B}_{R}, t \in \mathbb{N}_{\rho(a)}^{b}$ and consider

$$
\begin{aligned}
|(T y)(t)-(T z)(t)| & \leq \sum_{s=a+1}^{b}|G(t, s)||f(s, y(s))-f(s, z(s))| \\
& \leq K\|y-z\| \sum_{s=a+1}^{b}|G(t, s)| \\
& \leq \frac{K}{B(\alpha-1)}\left[(2-\alpha)+(\alpha-1) H_{\alpha-1}(b, a)\right]\|y-z\|,
\end{aligned}
$$

implying that $T$ is a contraction. Next, we show that $T\left(\partial \mathcal{B}_{R}\right) \subseteq \mathcal{B}_{R}$. To see this, let $y \in \partial \mathcal{B}_{R}, t \in \mathbb{N}_{\rho(a)}^{b}$ and consider

$$
\begin{aligned}
|(T y)(t)| & \leq \sum_{s=a+1}^{b}|G(t, s)||f(s, y(s))| \\
& \leq \sum_{s=a+1}^{b}|G(t, s)||f(s, y(s))-f(s, 0)|+\sum_{s=a+1}^{b}|G(t, s)||f(s, 0)| \\
& \leq K\|y\| \sum_{s=a+1}^{b}|G(t, s)|+P \sum_{s=a+1}^{b}|G(t, s)| \\
& \leq \frac{(K R+P)}{B(\alpha-1)}\left[(2-\alpha)+(\alpha-1) H_{\alpha-1}(b, a)\right] \leq R
\end{aligned}
$$

implying that $(T y) \in \mathcal{B}_{R}$. On the other hand, consider

$$
\begin{aligned}
|(T y)(t)| & \leq \sum_{s=a+1}^{b}|G(t, s)||f(s, y(s))| \\
& \leq Q \sum_{s=a+1}^{b}|G(t, s)| \\
& \leq \frac{Q}{B(\alpha-1)}\left[(2-\alpha)+(\alpha-1) H_{\alpha-1}(b, a)\right] \leq R
\end{aligned}
$$

implying that $(T y) \in \mathcal{B}_{R}$. Thus, we have $T\left(\partial \mathcal{B}_{R}\right) \subseteq \mathcal{B}_{R}$. Hence, (4.1) has a unique solution $y$ in $\mathcal{B}_{R}$ by Theorem 4.4. The proof is complete.

Theorem 4.7. (Global Existence \& Uniqueness) Assume $(C 1),(C$ 7) and $(C$ 8) hold. Then, (4.1) has a unique solution in $\mathbb{R}^{b-a+2}$. 
Proof. First, we claim that $T: \mathbb{R}^{b-a+2} \rightarrow \mathbb{R}^{b-a+2}$ is a contraction. To see this, let $y, z \in \mathbb{R}^{b-a+2}, t \in \mathbb{N}_{\rho(a)}^{b}$ and consider

$$
\begin{aligned}
|(T y)(t)-(T z)(t)| & \leq \sum_{s=a+1}^{b}|G(t, s)||f(s, y(s))-f(s, z(s))| \\
& \leq L\|y-z\| \sum_{s=a+1}^{b}|G(t, s)| \\
& \leq \frac{L}{B(\alpha-1)}\left[(2-\alpha)+(\alpha-1) H_{\alpha-1}(b, a)\right]\|y-z\|,
\end{aligned}
$$

implying that

$$
\|T y-T z\| \leq \frac{L}{B(\alpha-1)}\left[(2-\alpha)+(\alpha-1) H_{\alpha-1}(b, a)\right]\|y-z\| .
$$

Therefore, $T$ is a contraction. Hence, (4.1) has a unique solution $y$ in $\mathbb{R}^{b-a+2}$ by Theorem 4.5. The proof is complete.

\section{Examples}

Example 5.1. Consider the boundary value problem

$$
\left\{\begin{array}{l}
-\left(\begin{array}{l}
A B C \\
1
\end{array} \nabla^{1.5} y\right)(t)=t^{2}+(0.1) \sin y(t), \quad t \in \mathbb{N}_{2}^{9}, \\
y(0)=y(10)=0
\end{array}\right.
$$

Here $\alpha=1.5, f(t, y(t))=t^{2}+(0.1) \sin y(t), a=1$ and $b=10$. Clearly, $f(t, y)$ is continuous on $\mathbb{N}_{2}^{10} \times \mathbb{R}$. Further, $f(t, y)$ satisfies a Lipschitz condition with respect to $y$ on $\mathbb{N}_{2}^{10} \times \mathbb{R}$ with Lipschitz constant $L=0.1$. Consider

$$
\begin{aligned}
& \frac{L}{B(\alpha-1)}\left[(2-\alpha)+(\alpha-1) H_{\alpha-1}(b, a)\right] \\
& =\frac{(0.1)}{B(0.5)}\left[(0.5)+(0.5) H_{0.5}(10,1)\right] \\
& =\frac{(0.1)}{B(0.5)}[(0.5)+(0.5)(3.3386)]=\frac{0.2169}{B(0.5)} .
\end{aligned}
$$

If we choose $B(0.5)>0.2169$, then $\frac{L}{B(\alpha-1)}\left[(2-\alpha)+(\alpha-1) H_{\alpha-1}(b, a)\right]<1$, implying that (C 8 ) holds. Then, by Theorem 4.7 , the boundary value problem (5.1) has a unique solution $y$ in $\mathbb{R}^{11}$.

Example 5.2. Consider the boundary value problem

$$
\left\{\begin{array}{l}
-\left(\begin{array}{l}
A B C \\
1
\end{array} \nabla^{1.5} y\right)(t)=1+(0.1) y^{2}(t), \quad t \in \mathbb{N}_{2}^{9}, \\
y(0)=y(10)=0
\end{array}\right.
$$

Here $\alpha=1.5, f(t, y)=1+(0.1) y^{2}, a=1$ and $b=10$. Clearly, $f(t, y)$ is continuous on $\mathbb{N}_{2}^{10} \times \mathcal{B}_{R}$. Further, $f(t, y)$ satisfies a Lipschitz condition with respect to $y$ on $\mathbb{N}_{2}^{10} \times \mathcal{B}_{R}$ with Lipschitz constant $K=(0.2) R$. Also, we have

$$
P=\max _{t \in \mathbb{N}_{2}^{10}}|f(t, 0)|=1
$$


and

$$
Q=\max _{(t, y) \in \mathbb{N}_{2}^{10} \times \mathcal{B}_{R}}|f(t, y)|=1+(0.1) R^{2}
$$

Consider

$$
\begin{aligned}
& \frac{K}{B(\alpha-1)}\left[(2-\alpha)+(\alpha-1) H_{\alpha-1}(b, a)\right] \\
& =\frac{(0.2) R}{B(0.5)}\left[(0.5)+(0.5) H_{0.5}(10,1)\right] \\
& =\frac{(0.2) R}{B(0.5)}[(0.5)+(0.5)(3.3386)]=\frac{(0.4339) R}{B(0.5)} .
\end{aligned}
$$

Next, consider

$$
\begin{aligned}
& \frac{\frac{P}{B(\alpha-1)}\left[(2-\alpha)+(\alpha-1) H_{\alpha-1}(b, a)\right]}{1-\frac{K}{B(\alpha-1)}\left[(2-\alpha)+(\alpha-1) H_{\alpha-1}(b, a)\right]} \\
& =\frac{\frac{1}{B(0.5)}\left[(0.5)+(0.5) H_{0.5}(10,1)\right]}{1-\frac{(0.2) R}{B(0.5)}\left[(0.5)+(0.5) H_{0.5}(10,1)\right]} \\
& =\frac{\frac{(2.1693)}{B(0.5)}}{1-\frac{(0.4339) R}{B(0.5)}}=\frac{2.1693}{B(0.5)-(0.4339) R}
\end{aligned}
$$

Further, consider

$$
\begin{aligned}
& \frac{Q}{B(\alpha-1)}\left[(2-\alpha)+(\alpha-1) H_{\alpha-1}(b, a)\right] \\
& =\frac{\left[1+(0.1) R^{2}\right]}{B(0.5)}\left[(0.5)+(0.5) H_{0.5}(10,1)\right] \\
& =\frac{\left[1+(0.1) R^{2}\right]}{B(0.5)}[(0.5)+(0.5)(3.3386)]=\frac{(2.1693)\left[1+(0.1) R^{2}\right]}{B(0.5)} .
\end{aligned}
$$

To apply Theorem 4.6, we must have
(1) $\frac{(0.4339) R}{B(0.5)}<1$;
(2) $R \geq \frac{2.1693}{B(0.5)-(0.4339) R}$ or $R \geq \frac{(2.1693)\left[1+(0.1) R^{2}\right]}{B(0.5)}$.

If we choose $B(0.5)>1.38$, then $R=3$ satisfies the first and the second part of the second inequalities. Thus, by Theorem 4.6, the boundary value problem (5.2) has a unique solution $y$ in $\mathcal{B}_{3}$.

\section{Conclusion}

In this article, we derived a Lyapunov-type inequality for the boundary value problem (3.9) using the properties of the corresponding Green's function. Further, we also established sufficient conditions on existence and uniqueness of solutions for the boundary value problem (4.1) using Banach and Brouwer fixed point theorems. 


\section{References}

[1] T. Abdeljawad and D. Baleanu, Discrete fractional differences with non-singular discrete Mittag-Leffler kernels, Adv. Differ. Equ. 232 (2016).

[2] T. Abdeljawad and F. Madjidi, Lyapunov-type inequalities for fractional difference operators with discrete Mittag-Leffler kernel of order $2<\alpha<\frac{5}{2}$, Eur. Phys. J. Special Topics 226 (2017), 3355 - 3368.

[3] T. Abdeljawad, R. Mert and A. Peterson, Sturm-Liouville equations in the frame of fractional operators with exponential kernels and their discrete versions, Quaestiones Mathematicae 42 (2019), No. 9, 1 - 19.

[4] N. Acar, Development of nabla fractional calculus and a new Approach to data fitting in time dependent cancer therapeutic study, Masters Theses \& Specialist Projects, Report no. 1146, 2012.

[5] R. P. Agarwal, M. Meehan and D. O'Regan, Fixed point theory and applications, Cambridge Tracts in Mathematics, 141, Cambridge University Press, Cambridge, 2001.

[6] M. Bohner and A. Peterson, Dynamic equations on time scales: An introduction with applications, Birkhäuser Boston, Inc., Boston, MA, 2001.

[7] S. Charoenphon, Green's functions of discrete fractional calculus boundary value problems and an application of discrete fractional calculus to a pharmacokinetic model, Masters Theses \& Specialist Projects, Report no. 1328, 2014.

[8] R. A. C. Ferreira, A Lyapunov-type inequality for a fractional boundary value problem, Fract. Calc. Appl. Anal. 16 (2013), No. 4, 978-984.

[9] C. Goodrich and A. C. Peterson, Discrete fractional calculus, Springer, Cham, 2015.

[10] A. Ikram, Lyapunov inequalities for nabla Caputo boundary value problems, $J$. Difference Equ. Appl. 25 (2019), No. 6, 757-775.

[11] F. Jarad, Y. Adjabi, T. Abdeljawad, S. F. Mallak and H. Alrabaiah, Lyapunov type inequality in the frame of generalized Caputo derivatives, Discrete and Continuous Dynamical Systems Series S, DOI: 10.3934/dcdss.2020212.

[12] A. A. Kilbas, H. M. Srivastava and J. J. Trujillo, Theory and applications of fractional differential equations, Elsevier, Amsterdam, 2006.

[13] A. Liapounoff, Problème général de la stabilité du mouvement, Ann. Fac. Sci. Toulouse Sci. Math. Sci. Phys. (2) 9 (1907), 203 - 474.

[14] R. Mert, T. Abdeljawad and A. Peterson, A Sturm-Liouville approach for continuous and discrete Mittag-Leffler kernel fractional operators, Discrete and Continuous Dynamical Systems Series S, DOI: doi:10.3934/dcdss.2020171.

[15] D. M. Nguyen, Controllability and observability of the discrete fractional linear statespace model, Masters Theses \& Specialist Projects, Report no. 2574, 2018.

[16] B. G. Pachpatte, On Lyapunov type inequalities for certain higher order differential equations, J. Math. Anal. Appl. 195 (1995), No. 2, 527 - 536.

[17] I. Podlubny, Fractional differential equations, Academic Press, San Diego, 1999.

[18] S. Sengul, Discrete fractional calculus and its applications to tumor growth, Masters Theses \& Specialist Projects, Report no. 161, 2010.

[19] M. Uyanik, Analysis of discrete fractional operators and discrete fractional rheological models, Masters Theses \& Specialist Projects, Report no. 1491, 2015.

[20] F. Wu, Nabla fractional calculus and its application in analyzing tumor growth of cancer, Masters Theses \& Specialist Projects, Report no. 1217, 2012.

[21] G. Wu and D. Baleanu, Discrete fractional logistic map and its chaos, Nonlinear Dynam. 75 (2014), No. 1-2, 283-287.

Jagan Mohan Jonnalagadda 
Department of Mathematics, Birla Institute of Technology and Science Pilani, Hyderabad - 500078, Telangana, India.

E-mail address: $j \cdot j$ aganmohan@hotmail.com

Dumitru Baleanu

Department of Mathematics, Cankaya University, Ankara, Turkey.

Institute of Space Sciences, Magurele-Bucharest, Romania.

E-mail address: dumitru@cankaya.edu.tr

Received: April 14, 2020; Revised: July 3, 2020; Accepted: November 16, 2020 